\title{
Effects of tasks involving different numbers of processes on working memory: Assessment by conducting cognitive function tests and using NIRS oxygen monitoring equipment
}

\author{
Katsumi Sugihara ${ }^{1,3}$, Mizuho Fujiwara ${ }^{2}$, Hiroshi Furukawa ${ }^{2}$ \\ ${ }^{1}$ Faculty of Rehabilitation, Shijonawate Gakuen University \\ ${ }^{2}$ Faculty of Rehabilitation, Kobe Gakuin University \\ ${ }^{3}$ Graduate Student, Kobe Gakuin University Graduate School
}

\begin{abstract}
The present study examined the effects of tasks involving different numbers of processes on working memory (WM) by conducting cognitive function tests and using NIRS (near-infrared spectroscopy). The subjects were asked to perform two types of task involving varying numbers of processes. After the subjects performed both tasks involving one and multiple processes: drawing and the creation of a box, respectively, the oxygen hemoglobin concentration in the DLPFC was higher. However, there was no significant difference between the two tasks. The results of cognitive function tests following the production of boxes were also compared, and the PASAT 2 sec and SDMT scores were significantly higher. These results suggested that the efficient implementation of the task involving multiple processes generated an appropriate level of cognitive load required to promote the active functioning of WM and, as a result, the subjects achieved higher cognitive function test scores after completing the tasks.
\end{abstract}

Keywords: working memory, occupational therapy intervention, work-related practices, cognitive function

\section{Introduction}

Baddeley and Hitch [1] define working memory (WM) as a system designed to maintain temporarily information required for sets of complex cognitive processes, such as language understanding, learning, and inference, and to manipulate it. The function of $\mathrm{WM}$ is required to facilitate smooth actions by a person, and it principally operates in the dorsolateral prefrontal cortex (DLPFC). The prefrontal region not only serves as a basis for high-level cognitive functions, including thinking and cognition, but is also responsible for goal-oriented actions, such as performing behaviors and memorizing plans [2].

In recent years, an increasing number of studies have suggested that exercise activates the DLPFC. Ac-

Received: 6 October 2014, Accepted: 3 June 2015

Corresponding to: Katsumi Sugihara, Faculty of Rehabilitation, Shijonawate Gakuen University, 5-11-10, Hojo Daitou-city Osaka 5740011, Japan

e-mail: sugihara@reha.shijonawate-gakuen.ac.jp

C2016 Japanese Association of Occupational Therapists cording to Soya et al. [3], moderate or intense exercise for ten minutes activated the left DLPFC, and Taniguchi et al. [4] suggested that arm-reaching exercise activated the right DLPFC.

Other previous studies suggested that performing cognitive tasks improved cognitive functions and activated the prefrontal area. Komai et al. [5] asked patients with Alzheimer-type dementia to undergo training including visual and auditory tasks, and they received higher scores in cognitive function tests related to attentional function and WM following the training. Taniguchi et al. [4] conducted a test involving verbal WM tasks, and the prefrontal area of the subjects undergoing the tasks was activated, which suggested that WM functions are associated with the prefrontal area. According to the results of other previous studies, the implementation of meaningful tasks with respect to the values of individual participants [6] and antagonistic exercise performed by the elderly as a cognitive task effectively activated the prefrontal area [7]. Another previous study suggests that different tasks performed by examinees have varying influences on their cognitive functions. A study conducted by Nebes et al. [8] suggests that, al- 
though dysfunction cannot be noted when patients with mild Alzheimer-type dementia undergo simple attention tasks, patients tend to develop dysfunction while performing WM tasks and those that require the distribution and conversion of attention. These findings provided by previous studies suggest that performing exercise or cognitive tasks activates the cerebral blood flow in the prefrontal area and influences WM functions.

On the other hand, no previous studies focused on the number of processes involved in each task to examine the effects of its differences on WM. The system of WM actively processes information required to perform highly goal-oriented tasks while maintaining it [9]. Therefore, when a task involving multiple processes is performed, WM has a larger number of opportunities to maintain and operate information included in the processes actively. In other words, when a task involving a single process is performed, WM maintains and operates information fewer times. This led to the hypothesis that differences in the number of processes involved in each task may affect the activation of the DLPFC, which controls WM. The present study examined the effects of those differences on WM, using cognitive function tests and NIRS oxygen monitoring equipment (NIRO200NX, Hamamatsu Photonics). The subjects were asked to perform two tasks involving different numbers of processes.

\section{Subjects and methods}

\section{Subjects}

The subjects were ten college students, including four males and six females (mean age: $20.3 \pm 0.5$ years old). The subjects were asked to undergo the Edinburgh Handedness Inventory to confirm that their right arms are dominant.

\section{Tasks and methods}

In the present study, the following tasks were performed: "the creation of a box" involving four processes and "drawing using a mirror" consisting of one process.

The task of creating boxes using A4 craft paper has been introduced to many clinical settings in Japan since occupational therapists adopted it to assess problemsolving skills. The task can be easily introduced for the assessment of task implementation skills and cognitive functions as well as treatment in occupational therapy because tools and materials for the creation of boxes are readily available. The task also has meaning as treatment since the created boxes can be used in daily life.

In the experiment, the examiner presented a completed box to the subjects and explained the work procedure to them. The subjects were asked to create boxes while referring to a process chart including development views and a completed box, without receiving advice. The box, the length, width, and depth of which were 4, 4 , and $3 \mathrm{~cm}$, respectively, was created using craft paper. The tools used included pencils, rulers, scissors, and staplers. Four processes, or the following instructions, and development views were written on the process chart: (1) Draw a development view on craft paper, (2) Cut out the view with scissors, (3) Fold the paper into the shape of a box while ensuring that the margins of the paper for paste are facing in the proper direction, and (4) Overlap the margins and staple them.

In the drawing session, reflection drawing equipment (Takei Scientific Instruments Co., Ltd.), designed to assess the dexterity of the hands and learning skills, was used. There was only one process: the subjects drew a line between two star-shaped lines, using only a pencil. Although the numbers of processes and tools used were smaller, other conditions, including the provision of an explanation of the work procedure, presentation of the process chart, designation of tools, and responses to questions, were similar to those for the creation of boxes. Since the drawing task involved only one process: "Drawing a line with a pencil between two lines - an outline of a figure, reflected on a mirror", the subjects were required to refer to the process chart fewer times than for the task of creating a box.

\section{Cognitive function tests}

As cognitive function tests to assess WM, the Paced Auditory Serial Addition (PASAT) 1 and 2 second, Symbol Digit Modalities (SDMT), and Digit Span Tests (recital of numbers) were adopted. In the PASAT [10], participants listen to a $\mathrm{CD}$ and continue adding onedigit numbers that they have just been informed of in their head. The numbers are read out at an interval of one or two seconds. In the SDMT [10], participants write a number that corresponds to each of nine symbols according to a rule. The recital of numbers [10] assesses the auditory memory span, and there are two types: participants recite the numbers that have been read out by an examiner in the normal or reverse order (normal- and reverse-order recitals).

\section{Measuring equipment}

NIRS (near-infrared spectroscopy) is a non-invasive brain-function diagnostic imaging technique used to measure cerebral activities. In rehabilitation and other fields, oxygen hemoglobin (Oxy-Hb) concentrations and their changes, which depend on neural activity, have been measured [11]. The prefrontal area is considered to be a representative cortex in charge of performing WM tasks. In recent years, an increasing number of studies 


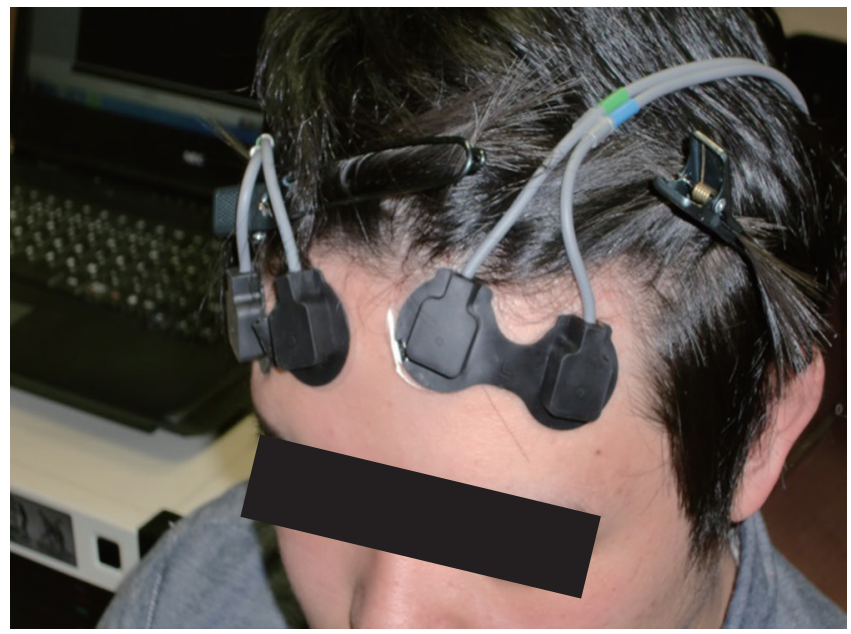

Fig. 1. Dipping of probes.

have been conducted to assess the functions of the frontal lobe using NIRS [2]. NIRS was adopted to analyze the activation of the DLPFC - the targeted measurement site. A pair of one-channel probes (for left and right) were attached $5 \mathrm{~cm}$ from the $\mathrm{C} 3$ and $\mathrm{C} 4$ points in the sagittal direction according to the international 10-20 method, based on the method developed by George et al. [12]. Time-series NIRS data within a specific period of time are related to each other, and cannot be regarded as independent. Therefore, a block design was adopted, in which the same task followed by a rest was repeated, to analyze statistically changes in the Oxy-Hb concentration during the tasks [13].

Furthermore, there were also spontaneous periodic variations in NIRS measurements other than those attributed to the activation of the brain while performing the tasks. In addition, the blood also flows in the tissues of the surface layer of the head other than the brain parenchyma, so NIRS measurements are also influenced by the dynamics of the blood flow. The above-mentioned results suggest that it is necessary to control the move- ment and postures of the subjects appropriately and implement baseline correction based on measurements at rest in order to prevent artifacts from interfering with NIRS measurements [14]. Therefore, prior to attaching the probes to the bodies of the subjects, they were asked to assume a specific sitting position so that their body movements could be controlled and their hands could be observed while performing the tasks. They were also instructed not to close or open their eyes too widely during measurement. After receiving these instructions, the subjects underwent measurement and performed the tasks when the waveform had become stable.

\section{Experimental procedures}

The experimental procedures are presented in Figs. 2 and 3. The experiment was conducted for each individual subject in an experimental room that lacked external stimuli. The following research procedure was implemented: (1) Cognitive functional tests (PASAT $1 \mathrm{sec}$ and $2 \mathrm{sec}$, SDMT, and recitals in the normal and reverse orders) had been conducted before the subjects performed the tasks. (2) NIRS probes were attached to the subjects and, after confirming that the waveforms were consistent, measurement was conducted while the subjects performed the tasks. (3) The subjects performed a 180 -second task four times at rest intervals of 30 seconds. While resting, the subjects were asked to assume the same posture as that when they performed the task, and gaze at the "+" mark on the screen of a PC. (4) When the subjects had completed the task, the probes were detached from them. (5) Cognitive function tests (PASAT $1 \mathrm{sec}$ and $2 \mathrm{sec}$, SDMT, and recitals in the normal and reverse orders) were conducted for the subjects who had completed the task.

The processes and tools were prescribed so that the subjects would not become confused when performing the task. The process of the task and tools were designated to help the subjects perform it efficiently and facilitate the active maintenance and manipulation of

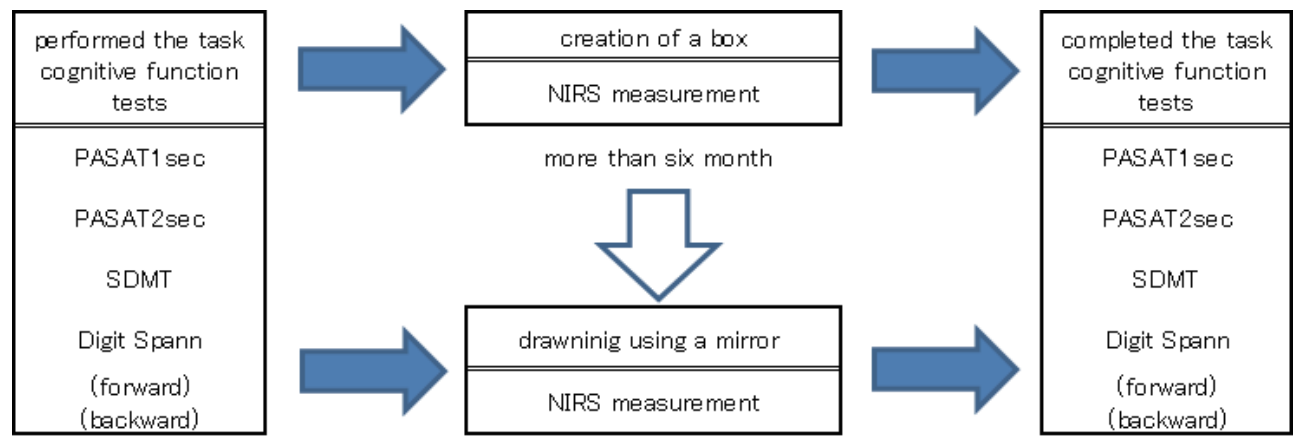

- The subjects perfomed the task of drawing more than six months after they had created boxes.

Fig. 2. Experiment procedures. 


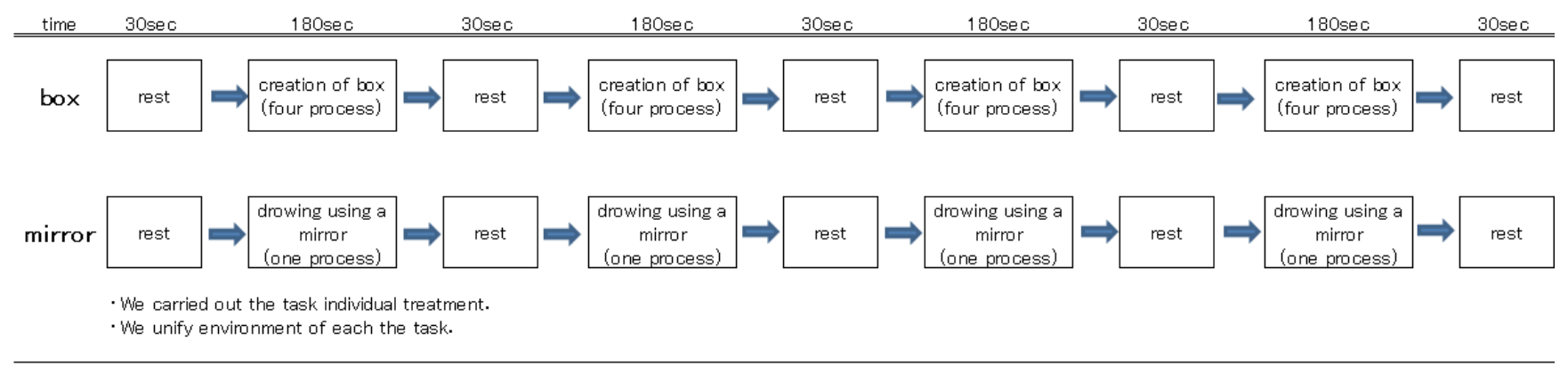

Fig. 3. Instruction procedures.

information - functions of WM. The examiner did not provide the subjects with advice on the method for performing the task efficiently. The subjects could improve the efficiency of performing the task - creation of four boxes, by implementing the following methods: omit drawing folding lines when "drawing a development view on craft paper", and reducing the frequencies of referring to the development view to confirm the dimensions and the process chart. Although the subjects were supposed to follow the order of the processes when performing the task, they were allowed to improve the work efficiency by modifying the procedure, which was expected to affect WM.

The subjects performed the task of drawing according to the same procedure more than six months after they had created boxes. This was because participation in cognitive function tests often enhances learning effects, and the experiments involving college students as the subjects were conducted during the periods of their summer and winter vacations. The subjects were in the same school year in the same college and involved in similar activities in their school lives. There was no significant difference in their mean age when they performed the tasks $(20.3 \pm 0.5$ and $21.1 \pm 0.3$ years old at the time of the production of boxes and drawing, respectively). The present study was conducted with the approval of the ethics committee of Kobe Gakuin University. The subjects received written and verbal explanations of the purpose of the study and its method, and consented to participate in it (Approval number: HEB120706-1).

\section{Analysis methods}

\section{Analysis of the activation of the DLPFC}

In NIRS data analysis, conducting averaging can isolate stimulus-dependent responses. Specifically, adding and averaging are performed to correspond to the intervals of similar stimuli applied multiple times to eliminate the waveforms of background activities, which fluctuate independently of the timing of the stimuli applied, and identify only stimulus-dependent responses [11]. In the present study, the body movements and postures were appropriately controlled to exclude data mixed with artifacts. The means of $\mathrm{Oxy}-\mathrm{Hb}$ concentrations during 170 of the 180 seconds required to perform each task, excluding the first 10 seconds, were calculated as the Oxy- $\mathrm{Hb}$ activation values, and their baseline correction was conducted. The mean of $\mathrm{Oxy}-\mathrm{Hb}$ concentrations measured during the first to third resting periods was calculated as the resting-state (baseline) value. The procedure for measurement was as follows: Immediately prior to the start of measurement, the subjects were instructed to look at a picture on a wall in front of them, and performed the tasks when the waveform had become stable.

The Wilcoxon signed-rank test was conducted to compare the $\mathrm{Oxy}-\mathrm{Hb}$ activation and resting-state values between the two tasks. The resting-state value was subtracted from the Oxy-Hb activation value for comparison, and the relative activation values and baseline levels were compared between the tasks, using the Mann-Whitney U test. The significance level was set to less than $5 \%$.

\section{Analysis of cognitive function test scores}

The subjects performed the task of drawing more than six months after they had created boxes because participation in cognitive function tests often enhances their learning effects. The cognitive function test scores before the tasks were performed were defined as the baseline values. The paired t-test was conducted to compare the scores received prior to and following the cognitive function tests between the two tasks. The baselines were compared between the two tasks, and differences between the cognitive function test scores received before and after the tasks had been performed were calculated and compared between the tasks, using Welch's test. The significance level was less than $5 \%$. 


\section{Results}

1. Oxy-Hb concentrations and baseline levels as the results of cognitive function tests

Table 1 shows that there were no significant differences in the Oxy-Hb concentration in the DLPFC and cognitive function test baselines for the tasks of creating boxes and drawing between the two tasks.

\section{Oxy-Hb concentrations}

(1) Resting-state and activation values (Oxy-Hb concentrations)

Table 2 shows Oxy-Hb concentrations (resting-state and activation values) measured during the creation of boxes and drawing.

1) Creation of boxes

Significant activation was noted in the right DLPFC (resting-state value in the right DLPFC: $1.0 \pm 1.0$ $\mu \mathrm{mol} / \mathrm{L}$, activation value in the right DLPFC: $1.7 \pm 1.5$ $\mu \mathrm{mol} / \mathrm{L}, p<0.05)$. There was no significant difference in the value in the left DLPFC (resting-state value in the left DLPFC: $0.7 \pm 1.0 \mu \mathrm{mol} / \mathrm{L}$, activation value in the right DLPFC: $1.3 \pm 1.7 \mu \mathrm{mol} / \mathrm{L})$.

\section{2) Drawing}

Significant activation was noted in both areas of the DLPFC (resting-state value in the left DLPFC: $0.2 \pm 1.4$ $\mu \mathrm{mol} / \mathrm{L}$, activation value in the left DLPFC: $0.9 \pm 1.5$ $\mu \mathrm{mol} / \mathrm{L}, \mathrm{p}<0.01$ ) (resting-state value in the right DLPFC: $1.5 \pm 0.9 \mu \mathrm{mol} / \mathrm{L}$, activation value in the right $\mathrm{DLPFC}$ : $2.6 \pm 1.1 \mu \mathrm{mol} / \mathrm{L}, p<0.01)$.
Table 2. Oxy-Hb concentrations (resting-state and activation values) measured during the creation of boxes and drawing.

\begin{tabular}{|c|c|c|c|c|c|c|c|}
\hline & \multirow[t]{2}{*}{ DLPFC } & & \multicolumn{2}{|c|}{$\begin{array}{l}\text { Resting- } \\
\text { state value } \\
(\mathrm{mmol} / \mathrm{L})\end{array}$} & \multicolumn{2}{|c|}{$\begin{array}{l}\text { Activation } \\
\text { value } \\
(\mathrm{mmol} / \mathrm{L})\end{array}$} & \multirow[t]{2}{*}{$\mathrm{P}$ value } \\
\hline & & & Mean & $\mathrm{SD}$ & Mean & $\mathrm{SD}$ & \\
\hline Box & Left & $\mathrm{Oxy}-\mathrm{Hb}$ & 0.7 & 1.0 & 1.3 & 1.7 & 0.2 \\
\hline Box & Right & Oxy-Hb & 1.0 & 1.0 & 1.7 & 1.5 & $0.04 *$ \\
\hline Mirror & Left & $\mathrm{Oxy}-\mathrm{Hb}$ & 0.2 & 1.4 & 0.9 & 1.5 & $0.0093 * *$ \\
\hline Mirror & Right & $\mathrm{Oxy}-\mathrm{Hb}$ & 1.5 & 0.9 & 2.6 & 1.1 & $0.0051 * *$ \\
\hline
\end{tabular}

$* * p<0.01 * p<0.05 \quad \mathrm{n}=10$

- The subjects performed the task of drawing more than six months after they had created boxes.

3) Comparison of relative $\mathrm{Oxy}-\mathrm{Hb}$ activation values between the tasks

Relative $\mathrm{Oxy}-\mathrm{Hb}$ activation values were compared between the tasks. There were no significant differences in the values for both sides between the two tasks (creation of a box in the left DLPFC: $0.6 \pm 1.1 \mu \mathrm{mol} / \mathrm{L}$, drawing in the left DLPFC: $0.6 \pm 0.6 \mu \mathrm{mol} / \mathrm{L})$ (creation of a box in the right DLPFC: $0.7 \pm 0.9 \mu \mathrm{mol} / \mathrm{L}$, drawing in the right DLPFC: $1.1 \pm 0.8 \mu \mathrm{mol} / \mathrm{L}$ ). Figs. 4 (creation of boxes) and 5 (drawing) show the mean waveforms for the two tasks.

Table 1. Oxy-Hb concentrations and baseline levels as the results of cognitive function tests.

\begin{tabular}{|c|c|c|c|c|c|c|c|c|}
\hline \multicolumn{3}{|c|}{ DLPFC } & \multicolumn{2}{|c|}{$\begin{array}{c}\text { The creation of } \\
\text { a box }\end{array}$} & \multicolumn{2}{|c|}{$\begin{array}{l}\text { Drawing using } \\
\text { a mirror }\end{array}$} & \multirow[t]{2}{*}{$\mathrm{U}$ value } & \multirow[t]{2}{*}{$\mathrm{P}$ value } \\
\hline & & & Mean & $\mathrm{SD}$ & Mean & SD & & \\
\hline Left & Oxy-Hb & $(\mathrm{mmol} / \mathrm{L})$ & 0.3 & 0.7 & 0.6 & 1.4 & 0.2 & 0.9 \\
\hline Right & $\mathrm{Oxy}-\mathrm{Hb}$ & $(\mathrm{mmol} / \mathrm{L})$ & 0.1 & 0.7 & 0.1 & 0.4 & 0.1 & 0.9 \\
\hline \multirow{2}{*}{\multicolumn{3}{|c|}{ Cognitive function tests }} & \multicolumn{2}{|c|}{$\begin{array}{c}\text { The creation of } \\
\text { a box }\end{array}$} & \multicolumn{2}{|c|}{$\begin{array}{l}\text { Drawing using } \\
\text { a mirror }\end{array}$} & & \multirow{2}{*}{$\mathrm{P}$ value } \\
\hline & & & Mean & $\mathrm{SD}$ & Mean & SD & & \\
\hline \multirow{5}{*}{\multicolumn{2}{|c|}{$\begin{array}{l}\text { PASAT1 second } \\
\text { PASAT2 second } \\
\text { SDMT } \\
\text { Forward } \\
\text { Backward }\end{array}$}} & (points) & 62.4 & 18.3 & 67.1 & 11.7 & & 0.5 \\
\hline & & (points) & 87.8 & 9.6 & 94.9 & 5.2 & & 0.1 \\
\hline & & (points) & 67.8 & 9.1 & 65.2 & 16.1 & & 0.7 \\
\hline & & (figure) & 7.1 & 0.9 & 7.0 & 1.1 & & 0.8 \\
\hline & & (figure) & 5.2 & 0.6 & 4.6 & 0.8 & & 0.2 \\
\hline
\end{tabular}

- The subjects performed the task of drawing more than six months after they had created boxes.

- Oxy-Hb: oxygen hemoglobin concentrations

- In the PASAT, participants listen to a CD and continue adding one-digit numbers that they have just been informed of in their head. The numbers are read out at an interval of one or two seconds.

- In the SDMT, participants write a number that corresponds to each of nine symbols according to a rule.

- The recital of numbers assesses the auditory memory span, and there are two types: participants recite the numbers that have been read out by an examiner in the normal or reverse order. 


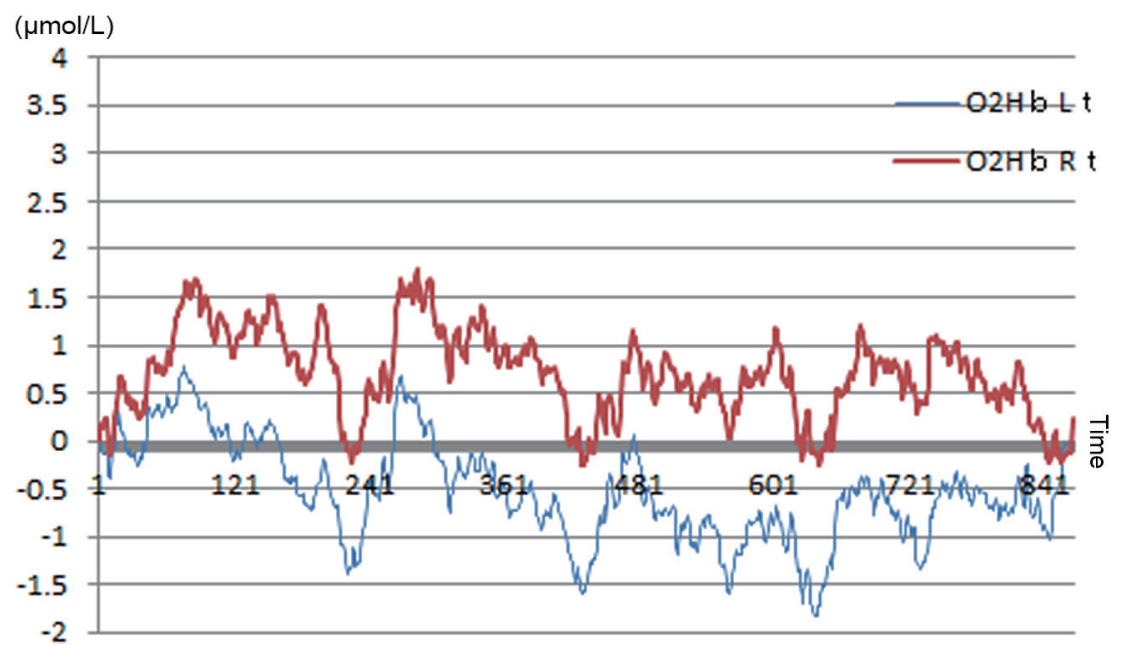

Fig. 4. Wave form of the creation of a box.

- Average waveform of relative value in the creation of a box.

- Bold line is the right side, and a thin line is a left side.

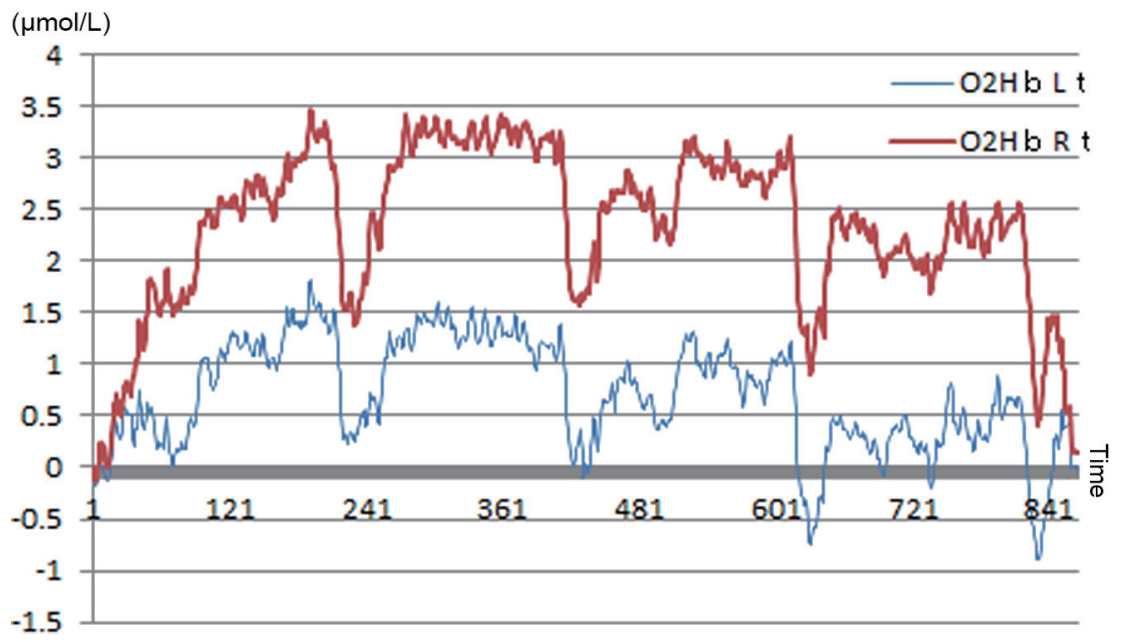

Fig. 5. Wave form of droawing using a mirror.

- Average waveform of relative value in droawing using a mirror.

- Bold line is the right side, and a thin line is a left side.

3. Results of cognitive function tests

(1) Comparison of cognitive function test scores before and after the tasks were performed

Table 3 shows the results of the comparison of cognitive function test scores before and after the creation of boxes and drawing were performed.

1) Creation of boxes

There were significant increases in the mean scores for the PASAT $2 \mathrm{sec}$ (prior to the task: $87.8 \pm 9.6$ points, following the task: $93.9 \pm 4.9$ points, $p<0.05$ ) and SDMT (prior to the task: $67.8 \pm 9.1$ points, following the task: $77.2 \pm 11.9$ points, $p<0.01$ ). There were no significant differences in the scores for the PASAT 1 sec (prior to the task: $62.4 \pm 18.3$ points, following the task: $67.9 \pm 13.8$ points), normal-order citation (prior to the task: $7.1 \pm 0.9$ points, following the task: $7.4 \pm 1.1$ points), and reverse-order citation (prior to the task: 5.2 \pm 0.6 points, following the task: $5.3 \pm 1.1$ points).

2) Drawing

There was a significant increase in the PASAT $1 \mathrm{sec}$ (prior to the task: $67.1 \pm 11.7$ points, following the task: $74.6 \pm 10.9$ points, $p<0.01$ ). There were no significant differences in the scores for the PASAT $2 \mathrm{sec}$ (prior to the task: $94.9 \pm 5.2$ points, following the task: $94.9 \pm$ 7.6 points), SDMT (prior to the task: $65.2 \pm 16.1$ points, following the task: $69.3 \pm 16.8$ points), normal-order citation (prior to the task: $7.0 \pm 1.1$ points, following the task: $7.2 \pm 1.0$ points), and reverse-order citation (prior 
Table 3. The results of the comparison of cognitive function test scores before and after the creation of boxes and drawing were performed.

\begin{tabular}{|c|c|c|c|c|c|c|}
\hline \multirow{2}{*}{\multicolumn{2}{|c|}{ The creation of a box }} & \multicolumn{2}{|c|}{ Prior to the task } & \multicolumn{2}{|c|}{ Following the task } & \multirow{3}{*}{$\begin{array}{c}\text { P value } \\
0.17\end{array}$} \\
\hline & & \multirow{2}{*}{$\begin{array}{c}\text { Mean } \\
62.4\end{array}$} & \multirow{2}{*}{$\frac{\text { SD }}{18.3}$} & \multirow{2}{*}{$\begin{array}{c}\text { Mean } \\
67.9\end{array}$} & \multirow{2}{*}{$\frac{\text { SD }}{13.8}$} & \\
\hline PASAT1 second & (points) & & & & & \\
\hline PASAT2 second & (points) & 87.8 & 9.6 & 93.9 & 4.9 & $0.02 *$ \\
\hline SDMT & (points) & 67.8 & 9.1 & 77.2 & 11.9 & $0.003 * *$ \\
\hline Forward & (figure) & 7.1 & 0.9 & 7.4 & 1.1 & 0.08 \\
\hline Backward & (figure) & 5.2 & 0.6 & 5.3 & 1.1 & 0.59 \\
\hline \multirow{2}{*}{\multicolumn{2}{|c|}{ Drawing using a mirror }} & \multicolumn{2}{|c|}{ Prior to the task } & \multicolumn{2}{|c|}{ Following the task } & \multirow{2}{*}{$P$ value } \\
\hline & & Mean & $\mathrm{SD}$ & Mean & $\mathrm{SD}$ & \\
\hline PASAT1 second & (points) & 67.1 & 11.7 & 74.6 & 10.9 & $0.004 * *$ \\
\hline PASAT2 second & (points) & 94.9 & 5.2 & 94.9 & 7.6 & 0.99 \\
\hline SDMT & (points) & 65.2 & 16.1 & 69.3 & 16.8 & 0.13 \\
\hline Forward & (figure) & 7.0 & 1.1 & 7.2 & 1.0 & 0.34 \\
\hline Backward & (figure) & 4.6 & 0.8 & 4.9 & 0.5 & 0.27 \\
\hline
\end{tabular}

to the task: $4.6 \pm 0.8$ points, following the task: $4.9 \pm 0.5$ points).

(2) Comparison of differences in cognitive function test scores prior to and following the tasks

Differences in test scores before and after the tasks of creating boxes and drawing had been performed (value following the task - value prior to it) were compared. There was a significant increase in the PASAT sec 2 score following the creation of boxes (creation of a box: $6.2 \pm 6.9$ points, drawing: $-0.01 \pm-4.7$ points, $p<0.05$ ). There were no significant differences in scores for cognitive function tests other than the PASAT $1 \mathrm{sec}$ (creation of a box: $5.4 \pm 11.3$ points, drawing: $7.5 \pm 6.1$ points), SDMT (creation of a box: $9.3 \pm 7.4$ points, drawing: $4.1 \pm 7.8$ points), normal-order citation (creation of a box: $0.3 \pm 0.4$ points, drawing: $0.2 \pm 0.6$ points), and reverse-order citation (creation of a box: $0.1 \pm 0.6$ points, drawing: $0.3 \pm 0.8$ points).

\section{Discussion}

In the present study, the subjects performed two tasks involving different numbers of processes, and the effects of their differences on WM were assessed by conducting cognitive function tests and NIRS, and based on the cerebral blood flow in the DLPFC and other test results. Prior to conducting analyses, the following point was confirmed: there were no significant differences in the Oxy-Hb concentrations in the DLPFC and baseline levels determined by the cognitive function tests between the two tasks.

The study results were as follows: there were no significant differences in the Oxy-Hb concentration in the DLPFC and baseline levels determined by the cognitive function tests after both tasks were performed. Although the Oxy-Hb concentration in the DLPFC was significantly higher after both tasks were performed, no significant difference was noted between the two tasks. Cognitive function test scores were significantly higher following the creation of a box, which involved multiple processes. There were significant increases in the mean scores for two cognitive function tests after the subjects performed the task of creating a box. In the comparison of cognitive function test scores between the two tasks, the mean score for the PASAT $2 \mathrm{sec}$ following the creation of a box was significantly higher.

$\mathrm{Oxy}-\mathrm{Hb}$ concentrations were significantly higher in the right area of the DLPFC after the subjects created a box, and in both left and right areas after they performed the task of drawing. An increase in the $\mathrm{Oxy}-\mathrm{Hb}$ concentration in the right prefrontal area is related to the positional relationship between a person and an object. On the other hand, an increase in the Oxy-Hb concentration in the left prefrontal area is related to the eye movement to gaze at an object and the function of WM to process spatial information $[15,16]$. There was a significant increase in the $\mathrm{Oxy}-\mathrm{Hb}$ concentration in the right prefrontal area following the creation of a box, presumably because WM continues using visual images when a subject creates a box. The task of drawing was performed using a mirror. Complex motor learning including the skillful use of a pencil by the subjects presumably involved the processing of information obtained from the eye movement and other visual infor- 
mation. These results suggest that the task of drawing significantly increased the $\mathrm{Oxy}-\mathrm{Hb}$ concentrations in both left and right prefrontal areas. However, there was no significant difference in the relative Oxy-Hb activation value between the two tasks. This suggests that the effects of tasks involving different numbers of processes on WM cannot be explained solely by an increase in the $\mathrm{Oxy}-\mathrm{Hb}$ concentration in the DLPFC.

Regarding the cognitive function tests following the completion of the tasks, the mean correct answer rates in the PASAT $2 \mathrm{sec}$ and SDMT were significantly higher after the subjects had created boxes. On the other hand, only the correct answer rate in the PASAT $1 \mathrm{sec}$ was significantly higher after the task of drawing was performed. In the comparison of the two tasks, the mean correct answer rate in the PASAT $2 \mathrm{sec}$ was significantly higher after the task of creating a box involving multiple processes had been performed, compared with the task of drawing. According to Callicott et al. [17], WM in the prefrontal area is less activated when the cognitive load is excessively large or small, and more activated when the appropriate amount of cognitive load is applied. The processes of the tasks and tools were prescribed to help the subjects concentrate on the tasks and to facilitate the active maintenance and manipulation of information functions of WM.

The number of processes involved in the creation of a box is larger than for the task of drawing, which means that there are more opportunities for improving the work efficiency. When the work efficiency was high, the appropriate amount of cognitive load was applied and WM was activated - there were significant changes in the cognitive function test scores following the completion of the tasks.

Levine et al. [18] conducted research on the effects of goal management training (GMT), and suggested that GMT, in which trainees learn about planning and goal accomplishment, is a method for improving frontal lobe dysfunction. In this method, the planning of a final goal involves the setting of multiple sub-goals, and the final goal is accomplished by conducting goal management, or the accomplishments of all sub-goals. Since WM functions the most effectively when people are goaloriented, undergoing GMT is expected to improve the functions of WM. When a box is created, or to reach the final goal, four processes as sub-goals are accomplished one by one and, as a result, WM is rewritten more frequently than when the task of drawing is performed. The goal of the task of drawing is to draw a line between two lines, which serve as an outline of a figure. When a line is drawn - a task involving only one process, subgoals are accomplished fewer times than when a box is created, and WM is also rewritten fewer times. WM is used less frequently during drawing than when a box is created, presumably due to a smaller number of processes required for the task of drawing. Kingberg et al. [19] suggest that, when patients undergo WM tasks as part of training, changing the difficulty level for each patient will improve the effect. The results of the present study suggest that, as the capacity of WM varies from person to person, WM can be effectively activated by changing the number of processes involved in tasks according to the WM capacity.

Although the present study focused on the number of processes involved in each task to examine the effects of its differences on WM, it is also necessary to discuss learning methods for performing tasks. When the subjects draw a line between two star-shaped lines reflected on a mirror, they make mistakes until they learn to use the pencil with dexterity. In other words, they make many mistakes to master complex motor learning and become able to draw properly. Since it is difficult to correct false responses caused by mistakes as one of the characteristics of the task, Watamori et al. [20] suggest that a learning method that prevents mistakes (errorless learning) effectively helps people memorize new things. In principle, the task of creating boxes is based on errorless learning because the process chart is referred to and it is not difficult to imagine a completed box. Both errorless learning and GMT are considered to be effective in the creation of a box involving four processes.

In the present study, the oxygen hemoglobin (Oxy$\mathrm{Hb}$ ) concentration in the DLPFC following the completion of the task was significantly higher, and cognitive function test scores were influenced by the creation of a box - a task involving multiple processes. However, there was no significant difference in the relative $\mathrm{Oxy}-\mathrm{Hb}$ activation value in the DLPFC between the two tasks. This suggests that, although the scores in the cognitive function test (designed to assess the functions of WM) following the production of boxes were higher than the test scores after the completion of the task of drawing, the effects of a difference in the number of processes on the functions of WM could not be adequately explained.

As a future task, it is necessary to measure the capacity of the WM of subjects, and calculate the appropriate amount of cognitive load applied when performing a task. The spatial resolution of NIRS is low and it can only determine values relative to $\mathrm{Oxy}-\mathrm{Hb}$ baseline values and their changes, so it will be necessary to interpret values by combining actual measurements with baseline values obtained using PET and other methods, as a future challenge. 


\section{Conclusion}

The present study examined the effects of two tasks involving different numbers of processes on WM by assessing the cerebral blood flow in the DLPFC and conducting cognitive function tests to assess the functions of working memory (WM). Although the oxygen hemoglobin $(\mathrm{Oxy}-\mathrm{Hb})$ concentration in the DLPFC was significantly higher after the subjects had performed both tasks, there was no significant difference between the two tasks. The results of cognitive function tests following the production of boxes were also compared, and the mean PASAT $2 \mathrm{sec}$ and SDMT scores were significantly higher. Cognitive function test scores after the two different tasks had been performed were compared, and the PASAT $2 \mathrm{sec}$ score following the task of creating a box was significantly higher than the score after drawing. The processes of the tasks and tools were prescribed to help the subjects perform the tasks efficiently and facilitate the active maintenance and manipulation of information - functions of WM. The task of creating a box, which involves a larger number of processes, provided the subjects with an opportunity for performing it more freely and efficiently than the task of drawing. The results suggested that the efficient implementation of the task involving multiple processes generated an appropriate level of cognitive load required to promote the active functioning of WM and, as a result, the subjects achieved higher cognitive function test scores after completing the tasks. The scores in the cognitive function test (designed to assess the functions of WM) following the production of boxes were higher than the test scores after the completion of the task of drawing, presumably because the production of a box involved multiple steps, in which the subjects were able to improve their work efficiency. However, since there was no significant difference in the relative $\mathrm{Oxy}-\mathrm{Hb}$ activation value between the two tasks, the effects of a difference in the number of processes have not yet been completely clarified.

Acknowledgement: I would like to express my sincere appreciation to the ten people who participated in the study.

\section{References}

[1] Baddeley A \& Hitch G. Working memory. In Bower, G.H., The psychology of learning and motivation. New York: Academic Press; 1974; 8: 47-89.

[2] Hase K. Rehabilitation treatments based on motor learning theory. (in Japanese) Tokyo: Ishiyaku Publishers, Inc.; 2008.

[3] Soya H, Hyodo H \& Sakatani K. (eds.) NIRS basics and clinical practice. (in Japanese) Tokyo: Shinko Igaku Shuppansha; 2012.

[4] Taniguchi H, Matsuo K, Maeoka H \& Morioka S. Neurorehabilitation and brain functional imaging 3 arm reaching exercise. (in Japanese) Journal of Physical Therapy. 2010; 27-4: 499-504.

[5] Komai Y \& Shigeta M. Effect of attention training on memory impairment in mild Alzheimer's disease patients. (in Japanese) Occupational Therapy. 2010; 29-4.

[6] Sawada T, Tachiki K, Fujita S \& Matsubara A. The influence of meaningful occupation on the prefrontal lobe. (in Japanese) The Journal of Japanese Association of Occupational Therapists. 2009; 28-4.

[7] Tabira T, Nakamura G, Iso N, Sagari A \& Hirase T. The development of antagonistic exercises as part of a cognitive program to prevent dementia in the elderly. (in Japanese) The Journal of Japanese Association of Occupational Therapists. 2012; 31-4.

[8] Nebes RD \& Brady CB. Focused and divided attention in Alzheimer's disesse. Cortex. 1989; 25(2): 305-15.

[9] Tanemura J. Clinical problems in rehabilitation of patients with dysexecutive syndrome. (in Japanese) Higher Brain Function Research. 2008; 28(3): 68-75.

[10] Japan Society for Higher Brain Dysfunction. Clinical assessment for attention and spontaneity. (in Japanese) Tokyo: Shinko Igaku Shuppansha; 2008.

[11] Takeda K. Signal processing for data of near-infrared spectroscopy. (in Japanese) Bulletin of International University of Health and Welfare. 2007; 12(2): 72-8.

[12] George MS, Lisaby SH \& Sackeim HA. Transcranial Magnetic Stimulation: applications in Neuropsychiatry. Arch gen Psychiatry. 1999; 56(4): 300-11.

[13] Sakatani K. NIRS Basics and Clinical Practice. (in Japanese) Tokyo: Shinko Igaku Shuppansha; 2012.

[14] Aizawa N, Uchiumi C, Nakamura Y, Makita K, Ishibashi M \& Iwakiri M. A literature review of the assessment of the dynamics of the blood flow in the frontal lobe using near-infrared spectroscopy (NIRS). - Focusing on cognition-related problems - School crisis and mental care. 2009; 2: 59-72.

[15] Taniguchi H \& Kawamura T. Neuro-rehabilitation and brain functional imaging 7 attention and working memory. (in Japanese) Journal of Physical Therapy. 2010; 27(8): 931-7.

[16] Smith EE, Jonides J, Koppe RA, Awh E, Schumacher EH \& Minoshima S. Spacial versus object working memory: Pet investigations J Cog Neurosci. 1995; 7: 337-56.

[17] Callicott JH, Mattay VS, Bertolino A, Finn K, Coppola R, Frank JA, Goldberg TE \& Weinberger DR. Physiological characteristics of capacity constraints in working memory as revealed by functional MRI. Cerebral Cortex Journal. 1999; 9(1): 20-6.

[18] Levine B, Robertson IH, Clare L, Carter G, Hong J, Wilson BA, Duncan J \& Stuss DT. Rehabilitation of executive function: an experimental-clinical validation of goal management training. Journal of the International Neuropsychological Society. 2000; 6: 299-312. 
[19] Kingberg T, Femell E, Olesen PJ, Johnson M, Gustafsson P, Dahlstrom K, Gillberg CG, Forssberg H \& Westerberg H. Computerized training of working memory in children with ADHD - a randomized, controlled trial. Journal of the American of child \& Adolescent Psychiarty. 2005;
44(2): 77-186.

[20] Watamori T \& Honda R. Rehabilitation Approaches to memory problems. (in Japanese) The Japanese Journal of Rehabilitation Medicine. 2005; 42(5): 313-9. 